\title{
A Palm Vein Recognition Approach by Multiple Convolutional Neural Network Models
}

\author{
Felix Olanrewaju Babalola ${ }^{1}$, Önsen Toygar ${ }^{2 *}, Y_{1}$ Itan Bitirim $^{3}$ \\ ${ }^{1}$ Eastern Mediterranean University, Faculty of Engineering, Departmant of Computer Engineering, Famagusta, North Cyprus, via Mersin 10, Turkey, (ORCID: 0000- \\ 0003-2731-0693), felix.babalola@emu.edu.tr \\ ${ }^{2 *}$ Eastern Mediterranean University, Faculty of Engineering, Departmant of Computer Engineering, Famagusta, North Cyprus, via Mersin 10, Turkey, (ORCID: 0000- \\ 0001-7402-9058), onsen.toygar@emu.edu.tr \\ ${ }^{3}$ Eastern Mediterranean University, Faculty of Engineering, Departmant of Computer Engineering, Famagusta, North Cyprus, via Mersin 10, Turkey, (ORCID: 0000- \\ 0002-1780-2806), yiltan.bitirim@emu.edu.tr
}

(International Symposium on Multidisciplinary Studies and Innovative Technologies (ISMSIT) 2021 - 21-23 October 2021)

(DOI: $10.31590 /$ ejosat.1016532)

ATIF/REFERENCE: Babalola, F. O., Toygar, Ö. \& Bitirim, Y. (2021). A Palm Vein Recognition Approach by Multiple Convolutional Neural Network Models. European Journal of Science and Technology, (29), 237-242.

\begin{abstract}
A palm vein recognition system is proposed in this paper. The efficiency of three convolutional neural network models (VGG16, VGG19 and AlexNet) in palm vein biometrics is compared and then this study proposes to fuse them with Decision-Level Fusion. These models employ the use of high number of filters during training which leads to very high computation time, therefore, the filters are reduced in this study to drastically reduce computation time while maintaining the efficiency of the models. The proposed method is tested on three datasets secured from FYO, PUT and VERA databases. The proposed system significantly increases the accuracy of the system in comparison with the individual models and achieves $99.06 \%, 99.83 \%$ and $99.26 \%$ on FYO, PUT and VERA datasets, respectively.
\end{abstract}

Keywords: Biometrics, Palm vein, VGG16, VGG19, AlexNet, Decision-Level Fusion.

\section{Çoklu Evrişimli Sinir Ağı Modelleri İle Bir Avuç İçi Damar Tanıma Yaklaşımı}

$\ddot{O} \mathbf{z}$

Bu makalede bir avuç içi damar tanıma sistemi önerilmiştir. Üç Evrişimli Sinir Ağı (CNN) modelinin (VGG16, VGG19 ve AlexNet) avuç içi damar biyometrisindeki etkisi karşılaştırılmış ve daha sonra bu modellerin Karar-Seviyesi Kaynaşımı kullanarak birleştirilmesi önerilmiştir. Bu modellerin eğitiminde çok fazla süzgeç kullanıldığı için hesaplama süresi çok yüksektir. Dolayısıyla, bu çalışmada, modellerin verimliliğini muhafaza ederken hesaplama süresini de büyük ölçüde azaltmak için modellerde kullanılan süzgeçler azaltılmıştır. Önerilen yöntem, FYO, PUT ve VERA isimli üç halka açık veritabanı kullanılarak test edilmiştir. Bireysel modellerle kıyaslandığında, önerilen yöntemin doğruluğu önemli ölçüde artmıştır ve FYO, PUT ve VERA veri kümeleri üzerinde sırasıyla \%99.06, $\% 99.8$ ve $\% 99.26$ başarı elde edilmiştir.

Anahtar Kelimeler: Biyometri, Avuç içi damarı, VGG16, VGG19, AlexNet, Karar-Seviyesi Kaynaşımı.

\footnotetext{
* Corresponding Author: onsen.toygar@emu.edu.tr
} 


\section{Introduction}

Human recognition systems, where physiological traits such as face, iris, palmprint, fingerprint, voice and gait are used, have become leading technologies worldwide for human biometric authentication. Subsequently, several research studies have been done in this area, such as standalone usage of physiological traits or combination of multiple traits, or combining physiological with a non-physiological trait [1]. Technological innovations for fast and effective acquisition of these biometric traits have also positively affected research in this area.

Furthermore, hand vein pattern on the palm, hand dorsal, wrist and fingers as biometric traits has also been well received. They are now among the most effective biometric traits where high data security is required.

Consequently, usage of palm vein as biometric attribute has received positive attention in recent years especially because of its innate anti-spoof quality and stability. These qualities make it almost impossible to show a fake human hand to a hand vein sensor because veins cannot be read from lifeless bodies. The acquisition process is also user friendly, it can be contactless to achieve higher acceptability. The images obtained at the data capture level are not occluded by external factors that are possible elsewhere on human body, such as accessories, make up or clothing.

In this paper, we combined three Convolutional Neural Networks (CNN) models (VGG16 [2], VGG19 [3] and AlexNet [4]) to build a palm vein recognition system. Three datasets (FYO database [1], PUT database [5], VERA database [6]) were used to examine and present the efficiency of the proposed system.

Contributions of this study are itemized below:

- Palm vein recognition with the fusion of three deep learning models.

- Faster CNN training with lesser filters.

- Comparative analysis of CNN models on palm vein recognition

- Decision-Level Fusion of CNN models.

The rest of this paper is outlined as follows: Section II gives a review of biometrics research with a focus on hand vein and palm vein biometrics. Section III presents a detailed description of the proposed system while Section IV shows the results of corresponding experiments. Section V presents the conclusion of the study and possible future works.

\section{Literature Review}

There has been several research works on the use of hand vein and other characteristics of the hand for human recognition, such hand geometry and shape [7], finger vein recognition [8] and dorsal vein recognition [9]. Capturing the vein pattern is the first stage of hand vein biometrics. Most studies in this field used images captured by near-infrared or infrared cameras [1], [10]. Similarly, numerous research have been carried out on how to extract features from sample hand vein images from the palm, dorsal, finger and wrist.

In 2011, Kabacinski and Kowalski introduced PUT database which is composed of a palm vein dataset along with a wrist vein dataset [5]. Additionally, Tome and Marcel introduced VERA palm vein database in 2015 , in order to use it to study print spoof e-ISSN: 2148-2683 attacks in hand vein biometrics [6]. Tongji Contactless Palmvein Dataset is a large scale palmvein database that was introduced in 2018. A deep CNN-based palmprint and palmvein recognition system was built using Tongji dataset [11].

Subsequently, several ways of using palm vein as a biometric trait have been proposed as well as how to effectively extract features from palm vein images. Identifying non-vein pixels by the extraction of line-based features of palm vein using directional filter bank has been used [12]. Maximal principal curvature algorithm with k-means algorithm has also been used for extraction from palm vein images [13]. On the other hand, ways to use palm vein practically have been proposed, such as ATM transaction security using palm vein biometric authentication combined with unique identification number [14]. Palm vein authentication technology and its application to financial solutions was introduced along with PalmSecure, an authentication product developed by Fujitsu for commercial usage [15].

Furthermore, Lee in 2012 constructed a camera-based device with NIR light source to obtain palm vein images and extracted features from the images using 2-D Gabor filter and VeinCode algorithm for fast template matching [16], while Adaptive Gabor filter was later used to improve the study [17]. Web camera and infrared LED illumination for low-cost hand vein acquisition has also been studied [18]. Multilayer security system with palm vein biometric using PCA and template matching techniques has also been proposed with an average accuracy of 92.00\% [19].

Combining palm vein with other physiological features or non-physiological features for stronger identification systems also exist in literature, such as palm vein features plus unique identification number for ATM security [14], combining palm, wrist and back of hand (dorsal) vein patterns. For example, a system was tested on deep learning based models and texturebased feature extraction methods [1]

\section{Proposed Method}

The proposed system is depicted with Fig. 1. The architecture is in three phases, namely pre-processing phase, model training phase and predictions combination phase. Detail of each phase is explained in the following subsections

\subsection{Pre-processing}

The only preprocessing required is obtaining the Region of Interest (ROI). This involves cropping out background areas and other extra areas, such as fingers and wrist, captured along with the palm vein image during the data acquisition process. In this study, the process of obtaining the ROI is carried out in a semiautomatic manner which involves first determining the best dimension that appropriately represents the ROI for all samples in the dataset. This is followed by automatic cropping of all the images using the determined dimension. These steps are repeated for images that will be badly cropped due to differences in hand size, orientation and hand position during data acquisition. The repetition is done until all images are appropriately cropped [10].

\subsection{CNN Models}

Three different CNN models were used in this study. The models are popular deep learning methods. However, these models make use of huge number of filters which are reduced in this study for faster computation time. The models, 
namelyVGG16, VGG19 and AlexNet, are explained below along with the changes made.

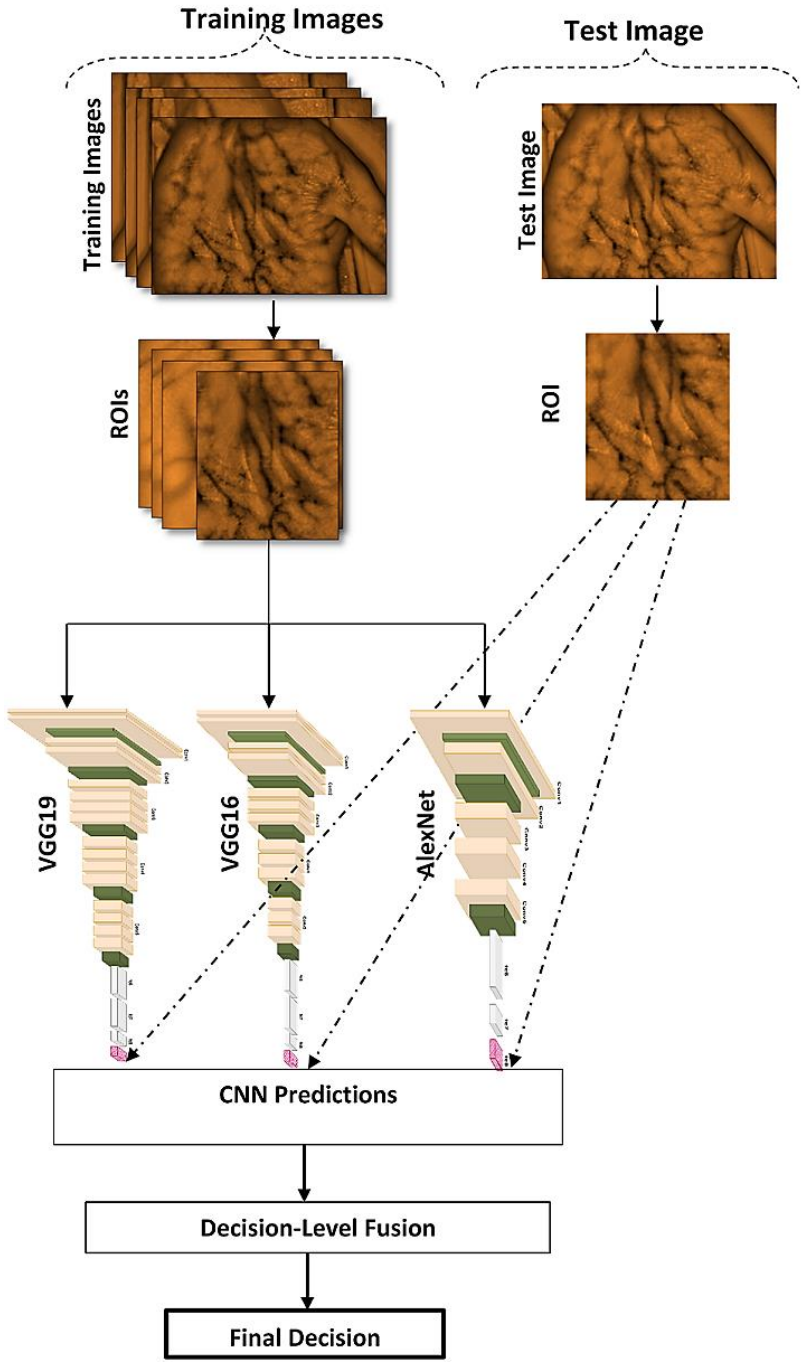

Fig. 1. Proposed system architecture

\subsubsection{VGG16}

VGG16 is a CNN model introduced in 2014 at the ILSVR (Imagenet) competition. The number 16 in the name represents the number of convolution layer in its structure. It has a large network, but is considered to be one of the successful CNN models. All convolutional layers use $3 \times 3$ filters and ReLU activation function. Although, there are 16 convolutional layers, there exist only five layers (two convolution layers are in the first layer, two in the second, three in the third, fourth and fifth layers, respectively), each followed by Batch Normalization layer and a Maxpool layer of $2 \times 2$ filter with two strides. The layers are completed with two Fully-connected layers, and a Softmax activation function which normalizes the output to be between 0 and 1.

Furthermore, each layer has high number of filters ranging from 64 to 512. However, as shown in Fig. 2, number of filters per layer was reduced in this experiment, making the training relatively small while still maintaining the efficiency of the models.

\subsubsection{VGG19}

Similar to VGG16, the number 19 in VGG19 represents the 19 convolutional layers in the model. VGG19 has all the features of VGG16 except that one additional convolutional layer is added to layers three, four and five as shown in Fig 3. It is also a highly successful CNN model with very large networks.

Table 1.VGG16 and VGG19 filter reduction percentages

\begin{tabular}{l|c|c|c}
\hline Layer & Original & Modified & $\begin{array}{c}\text { Reduction } \\
\text { percentage }\end{array}$ \\
\hline $\begin{array}{l}\text { First } \\
\text { layer }\end{array}$ & 64 & 32 & $50.00 \%$ \\
\hline $\begin{array}{l}\text { Second } \\
\text { layer }\end{array}$ & 128 & 64 & $50.00 \%$ \\
\hline $\begin{array}{l}\text { Third } \\
\text { layer }\end{array}$ & 256 & 96 & $62.50 \%$ \\
\hline $\begin{array}{l}\text { Fourth } \\
\text { layer }\end{array}$ & 512 & 128 & $75.00 \%$ \\
\hline $\begin{array}{l}\text { Fifth } \\
\text { layer }\end{array}$ & 512 & 128 & $75.00 \%$ \\
\hline
\end{tabular}

Fig. 2. VGG16 Architecture 


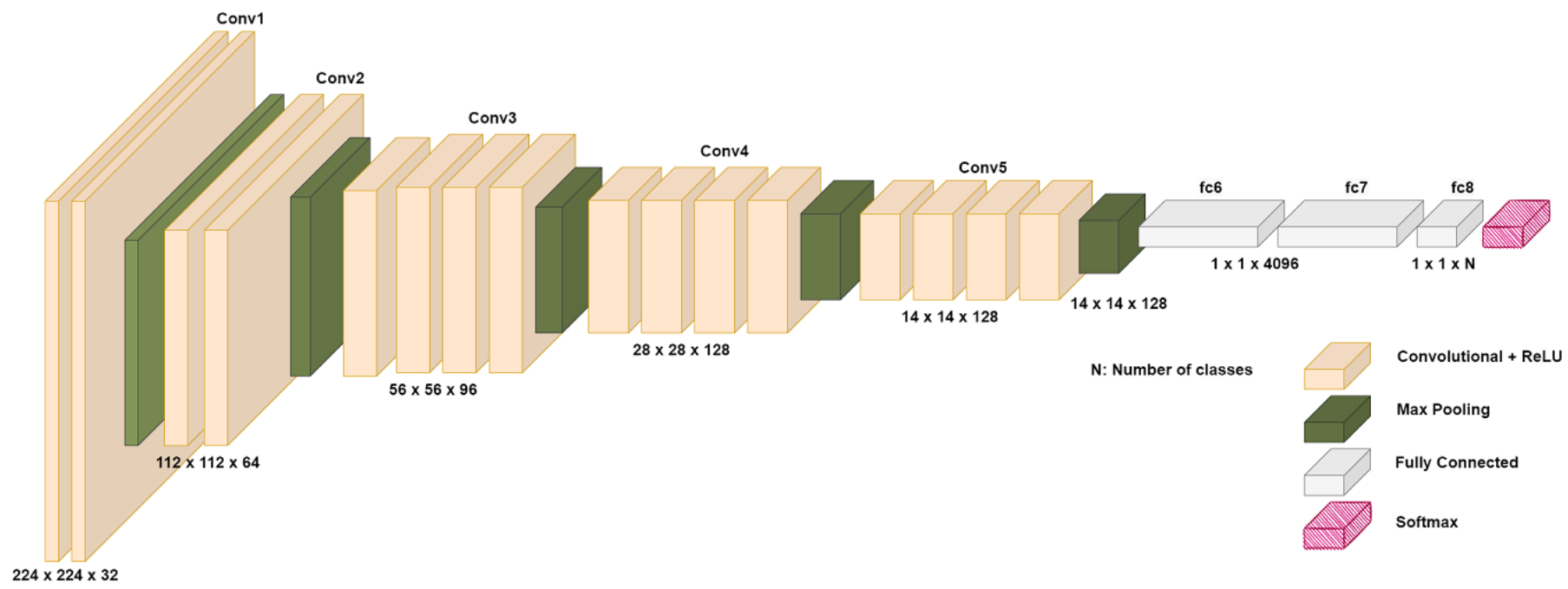

Fig. 3. VGG19 Architecture

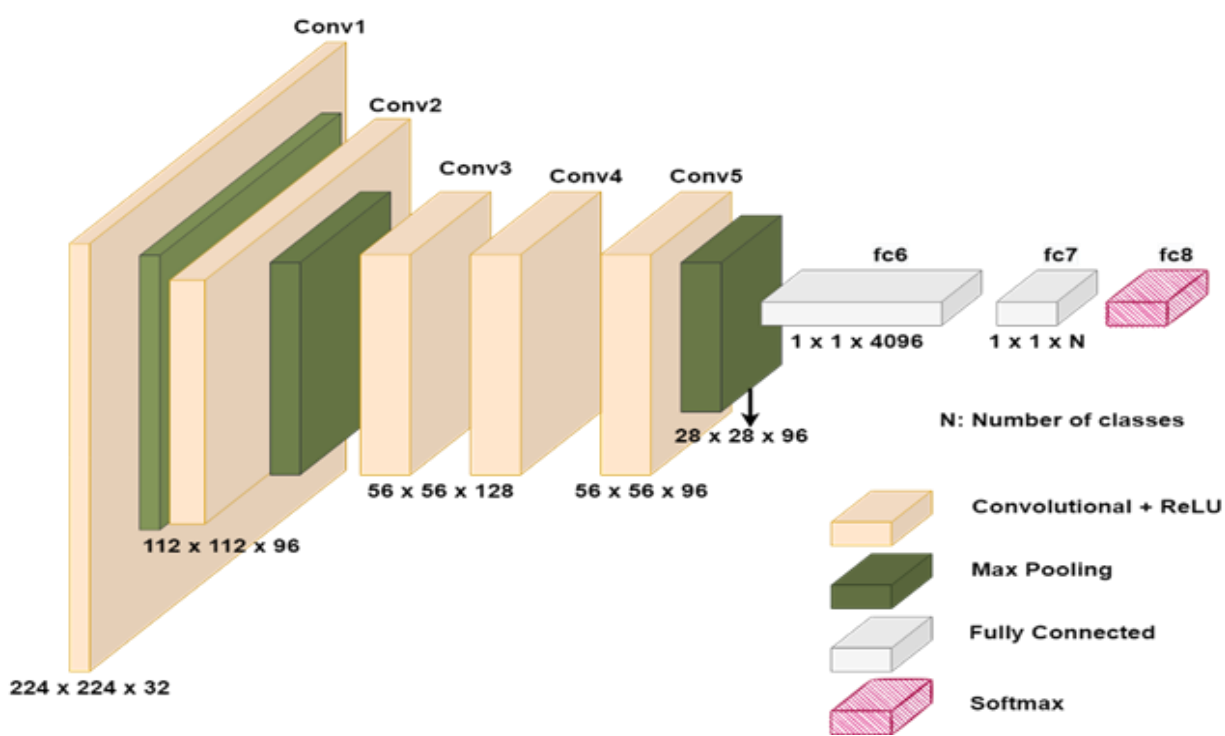

Fig. 4. AlexNet Architecture

Additionally, the same filter reduction in each convolutional layer was carried for VGG19 as in the aforementioned VGG16 experiment. Table 1 shows the differences in the number of filters in the original and modified VGG16 and VGG19 architectures used in this study.

Table 2. AlexNet filter reduction percentages

\begin{tabular}{l|c|c|c}
\hline Layer & Original & Modified & $\begin{array}{c}\text { Reduction } \\
\text { percentage }\end{array}$ \\
\hline $\begin{array}{l}\text { First } \\
\text { layer }\end{array}$ & $\begin{array}{c}11 \times 11 \times \\
96\end{array}$ & $3 \times 3 \times 32$ & $66.67 \%$ \\
\hline $\begin{array}{l}\text { Second } \\
\text { layer }\end{array}$ & $5 \times 5 \times 256$ & $3 \times 3 \times 64$ & $75.00 \%$ \\
\hline $\begin{array}{l}\text { Third } \\
\text { layer }\end{array}$ & $3 \times 3 \times 384$ & $3 \times 3 \times 128$ & $66.67 \%$ \\
\hline $\begin{array}{l}\text { Fourth } \\
\text { layer }\end{array}$ & $3 \times 3 \times 384$ & $3 \times 3 \times 128$ & $66.67 \%$ \\
\hline $\begin{array}{l}\text { Fifth } \\
\text { layer }\end{array}$ & $3 \times 3 \times 256$ & $3 \times 3 \times 64$ & $75.00 \%$ \\
\hline
\end{tabular}

\subsubsection{AlexNet}

AlexNet also has five layers but with just one convolutional layer in each. The original architecture has different filter sizes per layer, ranging from $3 \times 3$ to $11 \times 11$ as well as high number of filters, ranging from 96 to 384 . Each layer is followed by ReLU activation function, a Batch Normalization layer and a Maxpool layer of $3 \times 3$ filter. The architecture is completed with a $50 \%$ Dropout layer, Fullyconnected layer and Softmax activation function [4].

However, similar to VGG16 and VGG19, the number of filters was reduced in each layer as shown in Fig 4. Additionally, a 3 x 3 kernel was maintained for each convolutional layer throughout the architecture in this study. Table 2 shows the differences in the original architecture and the experimental model used in this study.

\subsection{Decision-Level Fusion}

The final decision of the proposed system is obtained by first obtaining individual decision from each of the three CNN models used; VGG16 (Decision I), VGG19 (Decision II) and AlexNet (Decision III). For each decision, a binary output of either correct or incorrect recognition is expected. Therefore, weight of 1 is 
Table 3. Computation time comparison (in seconds)

\begin{tabular}{l|c|c|c|c|c|c}
\hline Experiment & VGG16 & $\begin{array}{c}\text { Modified } \\
\text { VGG16 }\end{array}$ & VGG19 & $\begin{array}{c}\text { Modified } \\
\text { VGG19 }\end{array}$ & AlexNet & $\begin{array}{c}\text { Modified } \\
\text { AlexNet }\end{array}$ \\
\hline$F Y O$ & 131325.41 & 27843.18 & 136282.18 & 29402.61 & 109850.63 & 18446.27 \\
\hline$P U T$ & 107353.45 & 24838.32 & 123842.00 & 27353.93 & 141014.50 & 17300.00 \\
\hline$V E R A$ & 97840.38 & 22762.88 & 115968.66 & 24901.59 & 132259.94 & 15728.60 \\
\hline
\end{tabular}

assigned to correct recognition and 0 is assigned to incorrect recognition. All weights are added together per test sample. Consequently, a weight of 2 and 3 is assigned True in the final system decision, while a weight of 1 and 0 is assigned False.

\section{Experiments and Results}

\subsection{Datasets}

Palm vein datasets from FYO, PUT and VERA databases were used in this study. These datasets have different number of volunteers and different number of samples per person as described below.

FYO database [1] has multiple hand vein pattern datasets including dorsal vein, palmar vein and wrist vein. The number of volunteers used in the database is 160 . Hand vein images were captured from both hands of each volunteer, in two separate sessions. Consequently, the database has 640 images of palm vein patterns, as well as 640 images of dorsal vein and wrist vein patterns, respectively. However, only the palm dataset is used in this study.

Similarly, PUT vein database [5] is composed of both palm vein and wrist vein datasets. The number of volunteers in the database is 50 but samples were taken from both hands to make a total of 100 classes, as each hand is taken as a different entity. Consequently, a total of 1200 images taken in three separate sessions of four images per entity are in each dataset. Similarly, only palm vein dataset is used in this study.

VERA Palmvein Database [6] consists of 2200 images acquired from 110 volunteers among whom $63.64 \%$ were men and an age average of 33 years. Five images were taken from each hand of all volunteers in two sessions. Image capturing was performed in two separate locations; about $70 \%$ was captured in the first location, while the rest was captured in the second location.

\subsection{Data Preparation}

A large number of samples is necessary for training deep learning models in order to ensure proper training. Consequently, there is a need to increase the number of samples for all the datasets. Keras data generator which generates more images by altering input images' rotation, shear, width shift, height shift, zoom and brightness was used for this purpose [10]. This increased the number of samples to 6400 in FYO, 6000 in PUT and 5500 in VERA.

The test and training datasets were divided as follows:

- FYO: Training-set: 5760 (18 samples per entity), Testset: 640 (2 samples per entity)

- PUT: Training-set: 5400 (54 samples per entity), Testset: 600 (6 samples per entity)

- VERA: Training-set: 4950 (45 samples per entity), Testset: 550 (5 samples per entity).

\subsection{Experiment on Proposed System}

The resultant effect of reducing the number of filters per convolutional layer is the computation time which was reduced by approximately $450 \%$ with epochs for each training fixed at 30 . Training time for the datasets with the original and the modified models are presented in Table 3.

The results in Table 4 show that the models still perform well even with the high reduction in the amount of filters with approximately $96 \%$ average accuracy. On the other hand, the proposed method of combining the decisions of the three modified models outperformed the individual models, showing a more reliable model with $99.06 \%$ accuracy in FYO dataset, the $99.83 \%$ and $99.26 \%$ accuracy in PUT and VERA datasets, respectively.

Table 4. Experimental Results (Accuracy(\%))

\begin{tabular}{l|c|c|c}
\hline Experiment & FYO & PUT & VERA \\
\hline AlexNet & 90.53 & 94.33 & 98.53 \\
\hline VGG16 & 94.50 & 97.67 & 98.72 \\
\hline VGG19 & 96.09 & 97.67 & 94.50 \\
\hline $\begin{array}{l}\text { Proposed } \\
\text { method }\end{array}$ & 99.06 & 99.83 & 99.26 \\
\hline
\end{tabular}

\section{Conclusions and Recommendations}

This study introduces a palm vein recognition approach with the fusion of three deep learning algorithms. The study presented a comparison of the efficiency of three prominent CNN models (VGG16, VGG19 and AlexNet) in palm vein biometrics using three datasets, namely FYO, PUT and VERA databases. Comparable results were obtained across all three models. Additionally, Decision-Level Fusion of the three models was proposed in this study. The results showed significant increase in accuracy in the proposed system as $99.06 \%, 99.26 \%$ and $99.83 \%$ accuracy on FYO, VERA and PUT datasets, respectively. As a future work, subsequent research can be done on implementing similar person authentication with other hand vein traits including wrist vein, finger vein and dorsal vein. Other deep learning models as well as other algorithms for pattern recognition can also be used for palm vein biometrics.

\section{Acknowledge}

Three databases were very instrumental to the outcome of this study; FYO database which is available online at https://fyo.emu.edu.tr/en, PUT Vein Pattern Database was gotten from CIE Biometrics, while VERA Palmvein Database was gotten from Idiap Research Institute in Martigny and Haute Ecole Sp'ecialis'ee de Suisse Occidentale, Switzerland. 


\section{References}

[1]. Toygar, O., Babalola, F. \& Bitirim, Y. (2020). FYO: A Novel Multimodal Vein Database With Palmar, Dorsal and Wrist Biometrics. IEEE Access, 8, pp.82461-82470. https://doi.org/10.1109/ACCESS.2020.2991475.

[2]. Simonyan, K. \& Zisserman A. (2015). Very Deep Convolutional Networks for Large-scale Image Recognition. In Int. Conf. on Learning Representations (ICLR), San Diego, CA, USA, pp. 1-14.

[3]. Ha, I., Kim, H., Park, S. \& Kim, H. (2018). Image retrieval using BIM and features from pretrained VGG network for indoor localization. Building and Environment, 140, pp.2331. https://doi.org/10.1016/j.buildenv.2018.05.026.

[4]. Krizhevsky, A., Sutskever, I. \& Hinton, G. (2017). ImageNet classification with deep convolutional neural networks. Communications of the ACM, 60(6), pp.84-90. https://doi.org/ 10.1145/3065386.

[5]. Kabaciński, R. \& Kowalski, M. (2011). Vein pattern database and benchmark results. Electronics Letters, 47(20), p.1127.

[6]. Tome, P. \& Marcel, S. (2015). On the vulnerability of palm vein recognition to spoofing attacks. In: Proceedings of 2015 International Conference on Biometrics, ICB, pp. 319-325, https://doi.org/10.1109/ICB.2015.7139056.

[7]. Sharma, S., Dubey, S., Singh, S., Saxena, R. \& Singh, R. (2015). Identity verification using shape and geometry of human hands. Expert Systems with Applications, 42(2), pp.821-832. https://doi.org/10.1016/j.eswa.2014.08.052.

[8]. Sidiropoulos, G., Kiratsa, P., Chatzipetrou, P. \& Papakostas, G. (2021). Feature Extraction for Finger-Vein-Based Identity Recognition. Journal of Imaging, 7(5), p.89. https://doi.org/10.3390/jimaging7050089.

[9]. Nadiya, K. \& Gopi, V. P. (2020). Dorsal Hand Vein Biometric Recognition Based on Orientation of Local Binary Pattern. 2020 IEEE-HYDCON, pp. 1-6, https://doi.org/10.1109/HYDCON48903.2020.9242879.

[10]. Babalola, F., Bitirim, Y. \& Toygar, Ö. (2020). Palm vein recognition through fusion of texture-based and CNN-based methods. Signal, Image and Video Processing, 15(3), pp.459466.

[11]. Zhang, L., Cheng, Z., Shen, Y. \& Wang, D. (2018). Palmprint and Palmvein Recognition Based on DCNN and A New Large-Scale Contactless Palmvein Dataset. Symmetry, 10(4), p.78.

[12]. Wu, K., Lee, J., Lo, T., Chang, K. \& Chang, C. (2013). A secure palm vein recognition system. Journal of Systems and Software, 86(11), pp.2870-2876. https://doi.org/ 10.1016/j.jss.2013.06.065.

[13]. Wang, P. \& Sun, D. (2016). A research on palm vein recognition. 2016 IEEE 13th International Conference on Signal Processing (ICSP), pp. 1347-1351, https://doi.org/10.1109/ICSP.2016.7878046.

[14]. Prasanthi, B.V., Hussain, S. M., Kanakam, P. \& Chakravarthy, A. (2015). Palm Vein Biometric Technology: An Approach to Upgrade Security in ATM Transactions. International Journal of Computer Applications. 112, pp. 975-8887, https://doi.org/10.5120/19691-1440.

[15]. Watanabe, M., Endoh, T., Shiohara, M., \& Sasaki, S. (2005). Palm vein authentication technology and its applications. In: Proceedings of the Biometric Consortium Conference, pp. 37-38.
[16]. Lee, J. (2012). A novel biometric system based on palm vein image. Pattern Recognition Letters, 33(12), pp.15201528.

[17]. Han, W. \& Lee, J. (2012). Palm vein recognition using adaptive Gabor filter. Expert Systems with Applications, 39(18), pp.13225-13234.

[18]. Shah, G., Shirke, S., Sawant, S. \& Dandawate, Y. (2015). Palm vein pattern-based biometric recognition system. International Journal of Computer Applications in Technology, 51(2), p.105.

[19]. Athale, S., Patil, D., Deshpande, P. \& Dandawate, Y. (2015). Hardware Implementation of Palm Vein Biometric Modality for Access Control in Multilayered Security System. Procedia Computer Science, 58, pp.492-498. 